\title{
Ventromedial Prefrontal Cortex Lesions in Humans Eliminate Implicit Gender Stereotyping
}

\author{
Elizabeth Milne and Jordan Grafman \\ Cognitive Neuroscience Section, National Institute of Neurological Disorders and Stroke, National Institutes of Health, \\ Bethesda, Maryland 20892-1440
}

Patients with prefrontal cortex lesions and controls were administered an implicit association task (IAT) that measured the degree of association between male and female names and their stereotypical attributes of strength and weakness. They also completed three questionnaires measuring their explicit judgment regarding gender-related stereotypical attributes. There were no between-group differences on the explicit measures. On the IAT, patients with dorsolateral lesions and controls showed a strong association, whereas patients with ven- tromedial prefrontal cortex lesions had a significantly lower association, between the stereotypical attributes of men and women and their concepts of gender. This finding provides support for the hypothesis that patients with ventromedial prefrontal lesions have a deficit in automatically accessing certain aspects of overlearned associated social knowledge.

Key words: prefrontal cortex; social cognition; stereotypes; implicit association; social attitudes; ventromedial cortex
After ventromedial frontal lobe brain injury, patients often demonstrate acquired social conduct deficits such as an inability to respond appropriately to social cues in the environment or failure to obey conventional social rules. These deficits may be accompanied by a lack of awareness of what is socially appropriate (Eslinger and Damasio, 1985; Stuss et al., 1992; Damasio, 1995, 1996; Dimitrov et al., 1999). Because of these deficits, the ventromedial sectors of the prefrontal cortex have been considered the repository of social knowledge of the brain that is required for managing interpersonal interactions (Grafman, 1994, 1995). If this knowledge becomes inaccessible or degraded, behavior may default to inappropriate social rules for the situation or be guided by more instinctive or primitive responses induced by the environment (Grafman, 1994, 1995).

Evidence from previous studies has suggested that despite their aberrant real-life behavior, some patients with ventromedial frontal lobe lesions do possess, and can access, the social knowledge that they appear not to use in everyday life. For example, patient E.V.R.-frequently cited because of a striking dissociation between his preserved language, memory, and intellectual skills and his profoundly impaired social conduct, which developed after bilateral excision of orbital and lower mesial cortices-has been tested on several explicit measures of social cognition requiring means end problem solving of social situations (Platt and Spivack, 1974) and prediction of social solutions (O'Sullivan and Guilford, 1965) and was found to perform at levels equivalent or superior to normal controls (Saver and Damasio, 1991; Bechara et al., 1997). One explanation for his impaired social decision making (Bechara et al., 1997) is that ventromedial frontal lobe lesions result in a somatic marking deficit (Damasio, 1996, 1998). Somatic

\footnotetext{
Received Oct. 30, 2000; revised March 6, 2001; accepted March 28, 2001.

We thank Dr. Irene Litvan for her comments on earlier versions of this manuscript and Dr. Brian Fantie for helping to program the task.

Correspondence should be addressed to Dr. Jordan Grafman, Cognitive Neuroscience Section, National Institute of Neurological Disorders and Stroke, National Institutes of Health, Building 10/Room 5C205, MSC 1440, Bethesda, MD 208921440. E-mail: grafmanj@ninds.nih.gov.

Copyright (C) 2001 Society for Neuroscience $0270-6474 / 01 / 210001-06 \$ 15.00 / 0$
}

marking is a hypothesized mechanism that binds a positive or negative valence to a behavioral action that facilitates the decision of what is the correct or appropriate response in a given social situation.

On the other hand, Dimitrov et al. (1996) have demonstrated that unlike E.V.R., some patients with ventromedial lesions display impaired social knowledge compared with matched controls on an inventory designed to measure a person's understanding of the relative effectiveness of various solutions to everyday social problems (see also Dimitrov et al., 1999).

To further investigate the cause of the social conduct disorder frequently observed in patients with prefrontal cortex lesions, we adapted a task from the social cognition literature that addresses the nature and organization of stored social knowledge. The implicit association task (IAT) has been used to measure the automatic concept-attribute associations that are hypothesized to underlie implicit social attitudes and stereotypes (Greenwald and Banaji, 1995; Greenwald et al., 1998; Rudman and Kilianski, 2001). Normal subjects typically demonstrate relatively faster reaction times when they are asked to map stereotypically compatible (compared with incompatible) concepts onto a single response (known as the IAT effect). Scores on this implicit measure are often weakly (if at all) associated with explicit scores based on probing of attitudes or stereotypes (Greenwald et al., 1998). Stereotypes can be considered a form of social knowledge

This article is published in The Journal of Neuroscience, Rapid Communications Section, which publishes brief, peerreviewed papers online, not in print. Rapid Communications are posted online approximately one month earlier than they would appear if printed. They are listed in the Table of Contents of the next open issue of JNeurosci. Cite this article as: JNeurosci, 2001, 21:RC150 (1-6). The publication date is the date of posting online at www.jneurosci.org.

http://www.jneurosci.org/cgi/content/full/5330 
that is linked to actions, attitudes, rules, and other forms of knowledge and behavioral representation (Greenwald et al., 2000). This distributed representation constitutes a "social schema" (Carver et al., 1983). In a social schema, the various representations develop strong interconnections so that activation of one kind of representation can trigger activation of the others (leading to the IAT effect). It is likely that this automatic activation of the expression and comprehension of social knowledge or rules embodied in schemas occurs both in an associative manner between stored representations and across time associating individual schema events (Grafman, 1995). What is unknown is whether such associative social relations merely represents acquired social knowledge or, in addition, can influence or govern behavior in real social situations.

We decided to use the IAT to study the intactness of social knowledge in patients with prefrontal cortex damage. If aspects of social knowledge are stored in ventromedial prefrontal cortex, then damage to that cortical region should at least partially interfere with automatic associative relations between social knowledge attributes. To test this hypothesis, we studied normal volunteers and patients with ventromedial and dorsolateral prefrontal cortex damage and predicted that automatically activated social knowledge, typically demonstrated by normal subjects via the IAT effect, would be diminished in patients who suffer ventromedial compared with dorsolateral frontal lobe damage. In the version of the IAT we used, we examined subjects' sensitivity to the implicit association between concepts (male-female) and stereotyped attributes (weak-strong) related to gender.

\section{MATERIALS AND METHODS}

\section{Subjects}

We studied 10 male patients who were consecutively seen in our section and had suffered frontal lobe lesions (FLL) and 15 normal controls (NCs). The normal controls were all males who were screened to eliminate subjects who described a history of neurological or psychiatric disorder. All patients sustained a penetrating brain injury while serving in Vietnam from 1967-1970. The mean age of the normal controls was 52 ( \pm 14 years), and the mean age of the FLL patients was 52 ( \pm 2 years). The normal controls had a mean education level of 14.3 ( \pm 2 years) and an average full scale intelligence quotient (IQ) score of $113( \pm 6.6$ points). The FLL patients had a mean education level of $12.6( \pm 1.4$ years) and an average full scale IQ score of $92( \pm 10$ years $)$. There was no relationship between IQ scores and the IAT effect. Four of the FLL patients had a right unilateral lesion, two had a left unilateral lesion, and the four remaining patients had lesions that extended bilaterally. The FLL patients with ventromedial lesions had lesions that included Brodmann's areas 11, 12, 13, 14, or 47 based on an analysis of their CT scans using the Damasio and Damasio (1989) templates. The remaining three FLL patients with dorsolateral lesions had frontal lobe lesions that excluded Brodmann's areas 11, 12, 13, 14, or 47. The CT scan image analysis for volume loss was accomplished by using a light pen to outline the affected area across 24 brain slices (see Fig. 1 for a depiction of each patient's lesion). Total lesion volume was then calculated from a summation of these areas on relevant slices. The three dorsolateral prefrontal cortex lesion volumes fell within the $95 \%$ confidence interval of the ventromedial prefrontal cortex lesion volumes.

\section{Methods}

Implicit association test. Subjects were told they would be performing a word categorization experiment. All subjects were required to categorize male or female names and powerful or weak words (taken from Rudman and Kilianski, 2001). The stimuli consisted of 15 male names, 15 female names, 15 strong words, and 15 weak words.

Subjects were seated $\sim 0.5 \mathrm{~m}$ from the computer screen with the keyboard in front of them. They fixated on a rectangle $(150 \times 16 \mathrm{~mm})$ in the center of the screen within which the target word appeared. The rectangle was present on the screen at all times, and on either side of it were the category titles to remind subjects which side to press to make the correct response. The subjects' view is displayed in Table 1 (see below).

The target word remained on the screen until the subjects made their response. If the response was incorrect, the word "incorrect" appeared above the rectangle, although there was no opportunity to self-correct. After the end of each condition, subjects were debriefed to make sure that all the words were familiar to them.

Subjects responded by pressing one of two keys on the keyboard. All subjects were given the following verbal instructions for condition one, which were:

"In this task you have to categorize words into a particular category. A word will appear in this box here, and you must press one of these two keys in response. In this task if you see a male name you should press this key here on the left, and if you see a female name you press this key on the right. Please try to respond as quickly and as accurately as you can."

A similar instruction was given for the strong-weak words presented in conditions two and four. Before each condition began, it was clarified whether the subject would be categorizing gender names or strong-weak words. For conditions 3 and 5, when subjects responded to both gender names and attributes, they were told:

"Again you will see one word appear in this box. This time you have to press this key on the right if it is a male name or a word with connotations of strength and this key on the left if it is a female name or a word with connotations of weakness. Remember to be as accurate and as fast as you can.”

The IAT consisted of 210 total trials spread across the five conditions. As noted above, there were short breaks between the administration of each condition. Condition 1 required subjects to discriminate between male names and female names $(N=30$ trials). Conditions 2 and 4 required subjects to discriminate words from one of two stereotypical attributes $(N=30$ trials for each condition). Conditions 3 and 5 combine stimuli that are used in conditions 1,2, and 4 and involved mapping either a stereotypically associated attribute (e.g., female + weak-male + strong) or a stereotypically unrelated attribute (female + strong-male + weak) to the same hand ( $N=60$ trials for each condition). The key IAT result comes from a calculation of the difference in response times between conditions 3 and 5 . If the target categories are differentially associated with the attribute dimension, then the subject should find it easier to map concepts with their stereotypically associated attributes, which is reflected in relatively shorter response times. For example, if the subject associates women with weakness and men with strength, then their response times will be faster, for example, when they are mapping female names and weak words to the same response key than when they are required to map females names with strong words. The organization of conditions within the task is shown in Table 1.

Explicit scales. After finishing the IAT task, subjects completed three questionnaires that provided an explicit measurement of gender-related attitudes. The questionnaires were "The Attitude Toward Women Scale" (Spence and Helmreich, 1972), "The Ambivalent Sexism Inventory" (Glick and Fiske, 1996), and a semantic differential scale designed for this study, which consisted of eight bipolar adjectives selected from the words used in the IAT. Subjects were instructed to indicate where on the scale their conception of men and women (as independent constructs) would lie. Both patients and normal controls made remarks after completing the scales that indicated they felt their attitudes were going to be judged (e.g., "It's a good thing my wife isn't going to see what I put here"). On the other hand, during the IAT, both patients and normal controls thought they were simply categorizing words. Thus, based on this self report and the accumulated IAT literature, we did not expect a biasing effect of the IAT experience on the subjects' subsequent judgments on the explicit scales.

All subjects also completed a background history questionnaire in which they reported information about their upbringing (e.g., gender of siblings), education, work experience, work place environment, (malefemale dominated), marriage-divorce experience, and religion. This was to eliminate subjects who might have had a particularly unconventional background affecting their attitude toward, and interactions with, members of the opposite sex.

Statistical procedures. Response time and accuracy were recorded on each trial. Two-factor and one-way regular and repeated-measures ANOVAs, Kendall correlation coefficients, and Fisher's least significant difference (LSD) post hoc tests were used for statistical analyses of error rates and response times. The IAT effect is calculated by subtracting the mean response latency to the compatible stimuli from that of the incompatible stimuli. If the subject has a longer latency when responding to the 


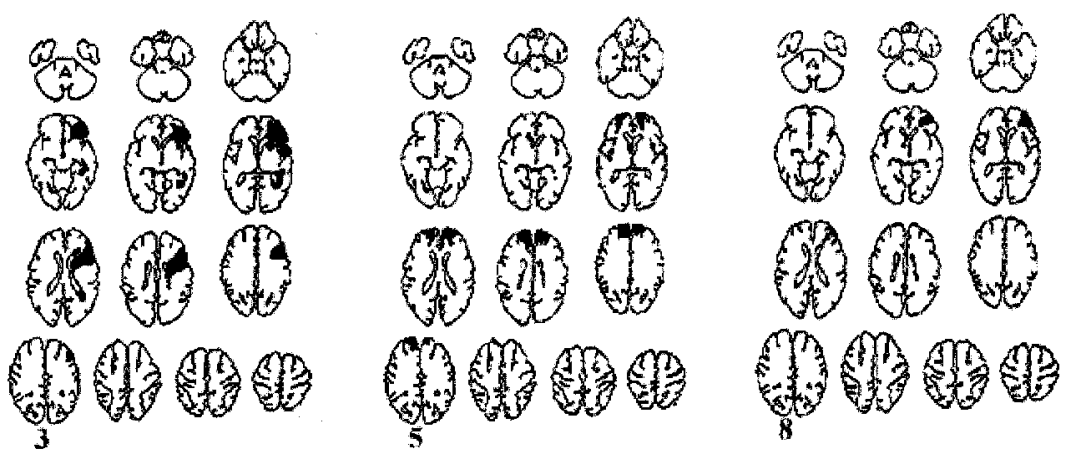

Patients without ventromedial damage

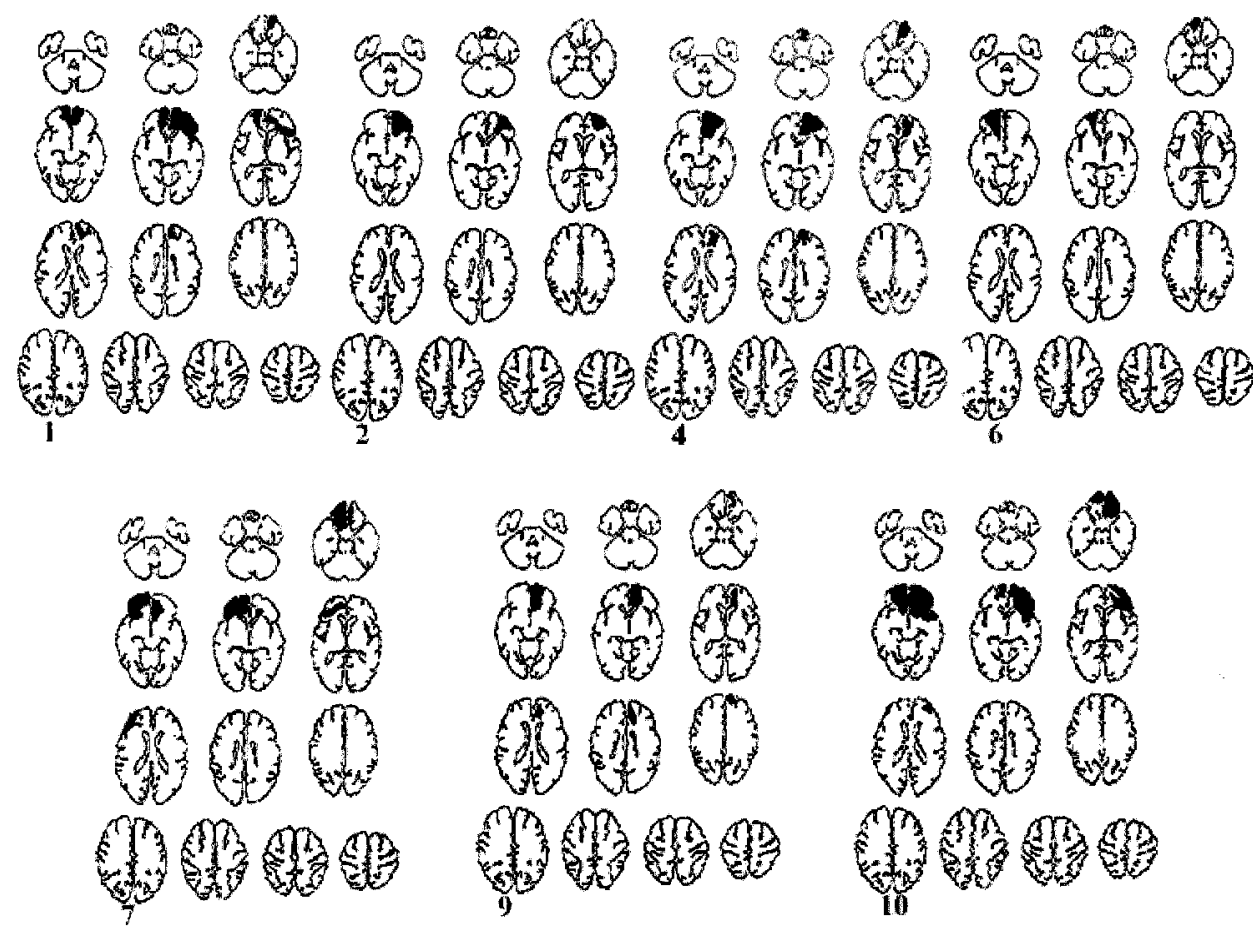

Patients with ventromedial damage

Figure 1. Templates of patient lesions. Patient numbers are indicated below each template.

incompatible stimuli, the effect is represented by a positive number (a higher value indicates a higher degree of association between the concept and its stereotypical attribute).

\section{RESULTS}

Error rates were typically low, with only slight variation between subject groups $(\mathrm{NCs}=4.4 \%$; patients $=7.7 \%)$. This is consistent with previous research (Greenwald et al., 1998) that has shown that error rates are less influenced than response time by compatibility.

For task 1 (involved categorizing names by gender), there was a marginally significant main effect for group on response times $\left(F_{(2,22)}=3.25 ; p=0.058\right)$ because of a significant difference in response times between NCs and patients without ventromedial lesions. For task 2 (involving strength and weakness words), there was a significant main effect $\left(F_{(2,22)}=36.28 ; p<0.0001\right)$ for group. Post hoc tests revealed between-group differences in response times between all groups. Finally, for task 4 (which was a repetition of task 2), there was again a significant main effect for $\operatorname{group}\left(F_{(2,22)}=12.93 ; p<0.0002\right)$. In this comparison, both NCs and patients with ventromedial lesions had significantly faster response times than patients without ventromedial lesions. There were no significant main effects for type of word or an interaction between word type and group.

We next looked at mean response times within subject to compare response times on the incompatible and compatible attribute trials. Normal controls showed mainly significant differences (using independent samples $t$ tests; $p<0.05$ ) between response times for the two conditions. The pattern of results for the patients was different however. We noticed that some patients did show a significant difference between their reaction time to compatible stimuli and their reaction time to incompatible stimuli, whereas others did not. On closer inspection it was discovered that patients with lesions outside the ventromedial prefrontal cortex (their lesion did not include Brodmann's areas 11, 12, 13, 


\begin{tabular}{|c|c|c|}
\hline \multirow{2}{*}{$\begin{array}{l}\text { Power/weakness IAT } \\
\text { Condition }\end{array}$} & \multicolumn{2}{|l|}{ Correct key press } \\
\hline & Left key & Right key \\
\hline 1 & Male name & Female name \\
\hline 2 & Strong word & Weak word \\
\hline 3 & Male name or strong word & Female name or weak word \\
\hline 4 & Weak word & Strong word \\
\hline 5 & Male name or weak word & Female name or strong word \\
\hline \multicolumn{3}{|c|}{$\begin{array}{l}\text { To counterbalance the order of presentation of stereotypical and nonstereotypical associations, half the subjects received an IAT block with } \\
\text { the following order: }\end{array}$} \\
\hline 1 & Male name & Female name \\
\hline 2 & Weak word & Strong word \\
\hline 3 & Male name or weak word & Female name or strong word \\
\hline 4 & Strong word & Weak word \\
\hline 5 & Male name or strong word & Female name or weak word \\
\hline \multicolumn{3}{|l|}{ Sample screen } \\
\hline \multirow[t]{2}{*}{ STRONG } & & WEAK \\
\hline & DOMINANT & \\
\hline
\end{tabular}

$\begin{aligned} & \text { Table 2. Response times to the compatible and incompatible stimuli } \\
& \text { and the mean IAT effect for all groups }\end{aligned}$
\begin{tabular}{llll} 
Subject group & $\begin{array}{l}\text { Mean RT } \\
\text { (comp.) (msec) }\end{array}$ & $\begin{array}{l}\text { Mean RT } \\
\text { (incom.) (msec) }\end{array}$ & $\begin{array}{l}\text { IAT effect } \\
\text { (msec) }\end{array}$ \\
\hline NC & 539 & 747 & 208 \\
SD & 96 & 178 & 131 \\
FLL w/VM & 684 & 779 & 95 \\
SD & 201 & 202 & 43 \\
FLL w-o/VM & 963 & 1303 & 340 \\
SD & 247 & 459 & 239
\end{tabular}

Response times for the compatible and incompatible conditions as well as for the IAT effect (comp, compatible; incom, incompatible).

14, and 47) appeared to demonstrate the same pattern as controls (significant difference between the compatible and incompatible trials), whereas patients with ventromedial prefrontal cortex lesions appeared to show little difference in their response times for the compatible and incompatible conditions. The subjects were therefore divided into three groups for further analysis (NCs, $n=$ 15), patients with ventromedial lesions (PVL, $n=7$ ), and patients without ventromedial lesions (PWVL, $n=3$ ). To validate this post hoc patient subgrouping, a one-way ANOVA was performed to measure the effect of order of trial presentation on IAT effect (see below). This was found to be nonsignificant.

A two-way ANOVA with a between-subjects factor of subject group (NC, PVL, and PWVL) and a within-subject factor of stimulus compatibility was used to investigate the interaction between the subject groups and the response times to the compatible and incompatible stimuli. For the mean reaction times of the different conditions, see Table 2.

There was a main effect of subject group $\left(F_{(2,22)}=9.28 ; p=\right.$ $0.0012)$ that occurred because the PWVL had significantly longer response time latencies than the other two groups, however this would not affect the critical within-group difference between response times to the compatible stimuli and incompatible stimuli. This was shown by a significant interaction between the subject groups and the compatibility of the stimulus $\left(F_{(2,22)}=\right.$ 4.021; $p=0.0325$ ). Post hoc analysis (Fisher's LSD test) indicated that the interaction was attributable to the NCs and the patients without ventromedial lesions both showing significant differences between their reaction times to the compatible and to the incompatible stimuli (response times being longer to incompatible stimuli), whereas the patients with ventromedial lesions did not show a significant difference between their reaction times to the different stimuli. This indicates that, like the NCs, patients with prefrontal cortex lesions that spare the ventromedial cortex show a response bias that facilitates responses to compatible stimuli and inhibits responses to incompatible stimuli. Patients who have lesions in the prefrontal cortex, which includes the ventromedial cortex, do not appear to show this response bias.

The effect was further investigated by calculating the mean IAT effect for each group of subjects. The IAT effect represents the difference in reaction time that people demonstrate when responding to compatible and incompatible trials. In line with Greenwald et al. (1998) this was calculated by subtracting the mean of the compatible trials from the mean of the incompatible trials for each subject individually. Table 2 shows the mean IAT effect for the three groups of subjects.

A one-way ANOVA of the mean IAT effects between the three groups showed a significant difference $\left(F_{(2,22)}=4.003 ; p=0.033\right)$, and the LSD post hoc test revealed that the patients with damage in the ventromedial prefrontal cortex had a significantly smaller IAT effect than the patients who had sustained damage to the dorsolateral prefrontal cortex $(p=0.012)$. The difference between the IAT effect of the patients with damage in the ventromedial prefrontal cortex and the NCs also approached significance $(p=0.073)$, whereas there was no significant difference between the mean IAT effect of the NCs and the patients without ventromedial damage $(p>0.1)$. In other words, NCs and patients without ventromedial lesions have a high IAT effect, indicating strong association between the target concept and its stereotypical attributes, whereas patients with a lesion in the ventromedial prefrontal cortex have a reduced IAT effect, suggesting they have a degraded associative linkage for this information.

To eliminate the possibility that performance was influenced by the size of the lesions, a linear regression was performed that showed no correlation between total brain volume loss and IAT 
effect $\left(r^{2}=0.03 ; p=0.63\right)$. In contrast to the group difference on the IAT, there were no between-group differences on the scales measuring explicit gender stereotyping. The scores on these scales indicated that, in general, the male subjects in this study endorsed statements indicating a moderate amount of gender stereotyping.

There were no between-patient group differences in test behavior nor were scores on a test examiner behavior rating scale significantly associated with the IAT effect. There was a modest correlation between the IAT effect and the total score on a Neuropsychiatric Symptom Inventory completed by the patient's significant other $(N=8$; Kendall $\tau=0.43)$, but this did not reach significance $(p=0.13)$. Data on the Neuropsychiatric Symptom Inventory was missing on two patients with ventromedial lesions who clearly had social behavior problems in real life based both on self report and direct observation.

\section{DISCUSSION}

Patients with ventromedial prefrontal cortex lesions showed impaired automatic priming of stereotypic social knowledge, whereas those with dorsolateral prefrontal cortex lesions performed like normal subjects. Social bias, such as a gender stereotype, represents social knowledge that is acquired during development. The content of the stereotype is dependent on cultural factors and the environmental influences to which an individual is subjected. Stereotypes reflect an economy of thought (Rosch, 1975), an attempt to organize the world around us into groups and categories that have certain perceived attributes (Rosch, 1975; Barsalou, 1992). As Bargh et al. (1996) has demonstrated, stereotypes can be triggered implicitly and automatically. This automatic elicitation of stereotypic social cognition, like that seen in associative lexical or object priming, probably reflects the organization of the underlying cognitive architecture of social knowledge and rules (that is based on the association between distinct social concepts or rules, between distinct social concepts or rules and other stimuli or actions, the frequency with which the social concept or rule is elicited, etc.). Despite the small number of subjects examined in our study, our results indicate that patients who have suffered ventromedial prefrontal cortex lesions have a degraded representation of social knowledge that can be demonstrated through priming experiments. Although inappropriate social conduct or knowledge in patients with ventromedial prefrontal cortex lesions is typically reported during real-time behavior, we were able to use a laboratory-based priming task to document a degraded social knowledge architecture. Given that there was no between-group difference in explicit judgments of stereotypes in this study, that finding suggests that a failure in the automatic processing of stereotypic social knowledge in patients with ventromedial prefrontal cortex lesions can sometimes be compensated for by an explicit conscious judgement or decisionparticularly if the patients believe their choices are being judged. In addition, there was a modest relation between the IAT effect and social behavior in real life, as measured by a significant other scale (the Neuropsychiatric Symptom Inventory). For the moment, we cannot generalize from these results and make claims about the intactness of other forms of social knowledge (besides gender attitudes) in patients with ventromedial lesions or whether these deficits in associative knowledge are specific to social knowledge (versus other forms of stimulus-response compatibility).

The response time latency for explicit social judgments such as those required on the inventories we used typically exceeds response times that are seen on tasks measuring implicit access to stored social knowledge and may be too delayed to appropriately reflect the rapid interpersonal processing required in certain social situations. Thus, using explicit measures of social cognition alone is probably insufficient to draw valid conclusions about the origin and nature of a social conduct disorder in brain-damaged patients.

Priming deficits, in general, are atypical for patients with frontal lobe lesions (Shimamura et al., 1992; Swick, 1998). For example, lexical priming has been viewed as spared after dorsolateral prefrontal cortex lesions (Swick, 1998). Although the IAT cannot be considered an equivalent task to repetition priming or other lexical priming tasks used in the cognitive literature, the IAT association trials are designed to facilitate activation of associative knowledge. In our study, gender bias was diminished in patients with ventromedial lesions. We believe that the representational structure of the underlying social-cognitive architectures and their interconnections were selectively impaired because we presume that they are stored within the ventromedial sectors of the prefrontal cortex. This would make priming among social knowledge associates within and across these architectures especially problematic. Although that explanation would predict a general deficit in accessing social knowledge, it is at odds with the results we obtained on the explicit scales that showed that patients with ventromedial lesions could demonstrate a typical amount of gender bias when endorsing written statements. A general deficit in decision making wouldn't easily account for our results either because both our implicit and explicit tasks required decision making. Rather, we must conclude that this dissociation suggests that the underlying neural systems used for rapid facilitation of associated social knowledge are distinct from those used for explicit recognition of social knowledge. Whereas a corollary to this observation exists in the literature on perceptual representation and processing [with perceptual processing of form dissociated from recognition processes (Fleischman et al., 1997)], we had assumed that the neural systems concerned with both explicit and implicit forms of social behavior would be represented within the ventromedial prefrontal cortex.

We also considered other, more trivial, explanations for our findings (e.g., fluctuations in attention or overall response speed). It is unlikely that a deficit in sustaining attention would have a specific effect in one condition versus another given the order in which conditions were presented and, in addition, there was no change in any subject's performance obvious to the tester that would have supported such an explanation. General betweengroup response time differences, furthermore, are unlikely to account for our results because both the fastest and slowest groups showed a similar IAT effect.

A recent functional MRI study by Phelps et al. (2000) included a version of the IAT that examined racial stereotypes and correlated performance on that behavioral task with the brain activity elicited while normal subjects were performing a face memory task based on race and found that amygdala activation was strongly associated with the IAT measure of race evaluation. Chee et al. (2000) used another version of the IAT that examined pleasant and unpleasant associations with various object categories to induce brain activation while normal subjects were performing the task. They found that left prefrontal cortex and anterior cingulate regions were activated in the incongruent condition of the task. These two studies indicate that different IAT measures and study designs may reveal different brain activation patterns. Thus, it is likely that the cortical representation of social cognition in humans will be distributed across several different 
neuronal networks, depending on the social behavior evaluated. In addition, our results, when combined with those of the study by Phelps et al. (2000), further suggest that the amygdala and prefrontal cortex are bound together as part of a neural network concerned with processing social cues and knowledge (Damasio, 1998). The amygdala may provide the somatic marker that then biases how social knowledge is retrieved or linked together (Damasio, 1996). Thus, another way to interpret the results of the present study is that ventromedial prefrontal cortex lesions interfere with somatic maker-social knowledge binding, which would primarily affect rapid automatic responses to social cues.

In summary, patients with ventromedial prefrontal cortex lesions show impaired automatic access to stereotypic social knowledge, whereas patients with dorsolateral prefrontal cortex lesions perform more like normal subjects. Because patients with ventromedial prefrontal cortex lesions are characterized by a lack of adherence to social rules and norms, it may be that a contributing factor to that social conduct impairment is the inability of those patients to automatically and rapidly associate differing aspects of social knowledge - a form of social agnosia.

\section{REFERENCES}

Bargh JA, Chen M, Burrows L (1996) Automaticity of social behavior: direct effects of trait construct and stereotype activation on action. J Pers Soc Psychol 71:230-244.

Barsalou LW (1992) Cognitive psychology: an overview for cognitive scientists. London: Erlbaum.

Bechara A, Damasio H, Tranel D, Damasio AR (1997) Deciding advantageously before knowing the advantageous strategy. Science 275:1293-1295.

Carver CS, Ganellen RJ, Froming WJ, Chambers W (1983) Modeling: an analysis in terms of category accessibility. J Exp Soc Psychol 19:403-421.

Chee MW, Sriram N, Soon CS, Lee KM (2000) Dorsolateral prefrontal cortex and the implicit association of concepts and attributes. NeuroReport 11:135-140.

Damasio AR (1995) On some functions of the human prefrontal cortex. Ann NY Acad Sci 769:241-251.

Damasio AR (1996) The somatic marker hypothesis and the possible functions of the prefrontal cortex. Philos Trans R Soc Lond B Biol Sci 351:1413-1420.

Damasio AR (1998) Emotion in the perspective of an integrated nervous system. Brain Res Brain Res Rev 26:83-86.

Damasio H, Damasio AR (1989) Lesion analysis in neuropsychology. New York: Oxford UP.
Dimitrov M, Grafman J, Hollnagel C (1996) The effects of frontal lobe damage on everyday problem solving. Cortex 32:357-366.

Dimitrov M, Phipps M, Zahn TP, Grafman J (1999) A thoroughly modern gage. Neurocase 5:345-354.

Eslinger PJ, Damasio AR (1985) A Severe disturbance of higher cognition after bilateral frontal ablation: patient E.V.R. Neurology 35:1731-1741.

Fleischman DA, Vaidya CJ, Lange KL, Gabrieli JD (1997) A dissociation between perceptual explicit and implicit memory processes. Brain Cogn 35:42-57.

Glick P, Fiske ST (1996) The Ambivalent Sexism Inventory: Differentiating hostile and benevolent sexism. J Pers Soc Psychol 69:1013-1027.

Grafman J (1994) Alternative frameworks for the conceptualization of prefrontal lobe functions. In: Handbook of neuropsychology (Boller F, Grafman J, eds), pp 187-202. Amsterdam: Elsevier.

Grafman J (1995) Similarities and distinctions among current models of prefrontal cortical functions. Ann NY Acad Sci 769:337-368.

Greenwald AG, Banaji MR (1995) Implicit social cognition: attitudes, self-esteem, and stereotypes. Psychol Rev 102:4-27.

Greenwald A, McGhee D, Schwartz J (1998) Measuring individual differences in implicit cognition: the implicit association test. J Pers Soc Psychol 74:1464-1480.

Greenwald AG, Banaji MR, Rudman LA, Farnham SD, Nosek BA, Rosier M (2000) Prologue to a unified theory of attitudes, stereotypes, and self-concept. In: Feeling and thinking: the role of affect in social cognition (Forgas JP, ed), pp 308-330. Paris: Cambridge UP.

O'Sullivan M, Guilford JP (1965) The measurement of social intelligence. Los Angeles: University of Southern California.

Phelps EA, O'Conner KJ, Cunningham WA, Funayama ES, Gatenby JC, Gore JC, Banaji MR (2000) Performance on indirect measures of race evaluation predicts amygdala activation. J Cogn Neurosci 12:729-738.

Platt JJ, Spivack G (1974) Means of solving real-life problems: I. Psychiatric patients versus controls and cross-cultural comparisons of normal females. J Commun Psychol 2:45-48.

Rosch E (1975) Cognitive representations of semantic categories. J Exp Psychol Gen 104:192-223.

Rudman LA, Kilianski SE (2001) Implicit and explicit attitudes towards female authority. Pers Soc Psychol Bull, in press.

Saver J, Damasio AR (1991) A preserved access and processing of social knowledge in a patient with acquired sociopathy due to ventromedial frontal damage. Neuropsychologia 29:1241-1249.

Shimamura A, Gershberg FB, Jurica PJ, Mangels JA, Knight RT (1992) Intact implicit memory in patients with frontal lobe lesions. Neuropsychologia 30:931-937.

Spence JT, Helmreich R (1972) The Attitudes Towards Women scale: an objective instrument to measure attitudes towards the rights and roles of women in contemporary society. In: JSAS Catalog of Selected Documents in Psychology, p 66a.

Stuss DT, Gow CA, Hetherington CR (1992) "No Longer Gage": frontal lobe dysfunction and emotional changes. J Consult Clin Psychol 60:349-359.

Swick D (1998) Effects of prefrontal lesions on lexical processing and repetition priming: an ERP study. Cogn Brain Res 7:143-157. 